\title{
Psychophysical and physiological responses to gratings with luminance and chromatic components of different spatial frequencies
}

\author{
Bonnie Cooper, ${ }^{1, *}$ Hao Sun, ${ }^{1}$ and Barry B. Lee ${ }^{1,2}$ \\ ${ }^{1}$ Graduate Center for Vision Research, SUNY College of Optometry, 33 West 42nd St., New York, New York 10036, USA \\ ${ }^{2}$ Max Planck Institute for Biophysical Chemistry, Göttingen, Germany \\ ${ }^{*}$ Corresponding author: bcooper@sunyopt.edu
}

Received September 12, 2011; revised December 7, 2011; accepted December 8, 2011; posted December 12, 2011 (Doc. ID 154496); published February 1, 2012

\begin{abstract}
Gratings that contain luminance and chromatic components of different spatial frequencies were used to study the segregation of signals in luminance and chromatic pathways. Psychophysical detection and discrimination thresholds to these compound gratings, with luminance and chromatic components of the one either half or double the spatial frequency of the other, were measured in human observers. Spatial frequency tuning curves for detection of compound gratings followed the envelope of those for luminance and chromatic gratings. Different grating types were discriminable at detection threshold. Fourier analysis of physiological responses of macaque retinal ganglion cells to compound waveforms showed chromatic information to be restricted to the parvocellular pathway and luminance information to the magnocellular pathway. Taken together, the human psychophysical and macaque physiological data support the strict segregation of luminance and chromatic information in independent channels, with the magnocellular and parvocellular pathways, respectively, serving as likely the physiological substrates. (C) 2012 Optical Society of America

OCIS codes: $\quad 330.1720,330.1800,330.1880,330.5380,330.5510$
\end{abstract}

\section{INTRODUCTION}

Natural scenes contain both luminance and chromatic content [1,2] with often complex spatial structure []]. There are two views as to how luminance and chromatic information in complex scenes might be conveyed. One hypothesis suggests that strict segregation of luminance and chromatic information in afferent channels is an optimal way to transmit information. Since peak absorbances of the middle- $(M)$ and longwavelength $(L)$ cones lie close to one another in the spectrum, early coding of $|L+M|$ and $|L-M|$ signals in ganglion cells reduces redundancy in signals leaving the retina and has other advantages [4-7]. However, another possibility is that there is substantial overlap in the transmission of luminance and chromatic signals multiplexed within the parvocellular (PC) pathway. This asserts that the PC pathway performs "double-duty" by carrying both luminance and chromatic content [8-10]. It has been suggested that multiplexing the visual signal conserves bandwidth while leaving the business of segregating luminance and chromatic information to higher visual centers [11].

The primate visual system contains separate systems with distinctive anatomical and physiological characteristics [12-14]. The physiological responses of PC, magnocellular (MC), and koniocellular (KC) retinal ganglion cells to luminance and chromatic contrast are distinctive and well characterized. $\mathrm{PC}$ and $\mathrm{KC}$ responses are spectrally opponent whereas MC responses exhibit a spectral sensitivity corresponding to the psychophysical luminosity function $V_{\lambda}$ and are thought to underlie performance on behavioral tasks such as heterochromatic flicker photometry [15]. Specific anatomical substrates for PC, MC, and KC pathways have been iden- tified [16]. The PC pathway contains cells with opponent middle- $(M)$ and long-wavelength $(L)$ cone input combined in a subtractive manner $(|M-L|)$, whereas MC cells receive the summed input of $M$ and $L$ cones $(|M+L|)$. The KC pathway, although the underlying circuitry is not as well delineated as that of the PC \& MC pathways, receives excitatory (or inhibitory) $S$-cone input opposed by some combination of the other cone types $[14,17]$.

Use of gratings combining two (or more) spatial frequencies has provided evidence for multiple channels in achromatic spatial vision $[18,19]$. Here we use gratings with chromatic and luminance components of different spatial frequencies; this is of particular interest because it may approximate more naturalistic stimuli. A recent approach has described responses of primate ganglion cells to such compound luminance and chromatic gratings in comparison to psychophysical performance [20]. We report here on an extension of this approach, again comparing human psychophysical performance with macaque ganglion cell responses. We expand the results with additional grating variants and a more comprehensive investigation of psychophysical performance with these gratings.

Figure 1 shows the stimuli used. Figures $1 \mathrm{~A}$ and $1 \mathrm{~B}$ show standard luminance and chromatic gratings, and Figs. $1 \mathrm{C}$ and 1D show gratings in which the chromatic grating component has half or twice the spatial frequency of the luminance component. The equations describing these gratings are as follows:

$$
\begin{aligned}
& R(\theta)=C_{\text {lum }} \sin (\theta)+1 \\
& G(\theta)=C_{\text {lum }} \sin (\theta)+1,
\end{aligned}
$$


A

B

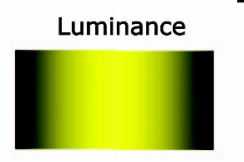

Chromatic
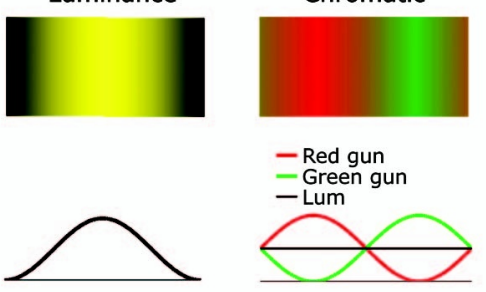

C

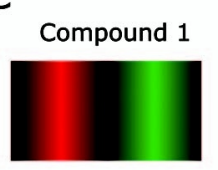

D
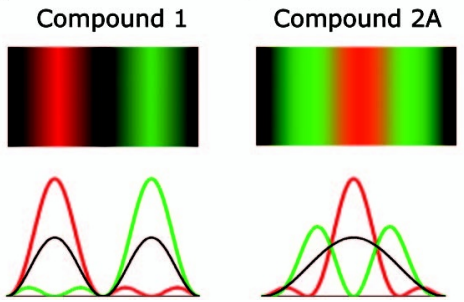

E

Compound 2B
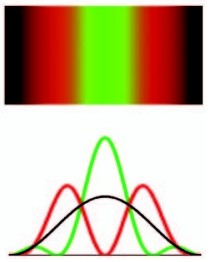

Fig. 1. Grating stimuli. (A) Luminance grating. (B) Isoluminant redgreen grating. (C) Compound 1, chromatic red/green modulation combined with luminance modulation of twice the spatial frequency; (D, E) Compound grating 2, chromatic red/green modulation of doubled spatial frequency is combined with a luminance grating. Two phase relations are shown (2A, 2B). Below each stimulus sample are waveforms representing the gun modulations (red and green); the solid black curve is the mean luminance of the two guns.

$$
\begin{aligned}
& R(\theta)=C_{\mathrm{chr}} \sin (\theta)+1 \\
& G(\theta)=-C_{\mathrm{chr}} \sin (\theta)+1,
\end{aligned}
$$

$$
\begin{aligned}
& R(\theta)=\left(-C_{\mathrm{lum}} \cos (2 \theta)+1\right)\left(C_{\mathrm{chr}} \sin (\theta)+1\right) / 2 \\
& G(\theta)=\left(-C_{\mathrm{lum}} \cos (2 \theta)+1\right)\left(-C_{\mathrm{chr}} \sin (\theta)+1\right) / 2,
\end{aligned}
$$

$$
\begin{aligned}
& R(\theta)=\left(C_{\text {lum }} \sin (\theta+\pi / 4)+1\right)\left(C_{\mathrm{chr}} \sin (2 \theta)+1\right) / 2 \\
& G(\theta)=\left(C_{\text {lum }} \sin (\theta+\pi / 4)+1\right)\left(-C_{\mathrm{chr}} \sin (2 \theta)+1\right) / 2,
\end{aligned}
$$

where $R(\theta)$ and $G(\theta)$ refer to the red and green gun modulations. Equations (1) and (2) refer to standard luminance and chromatic gratings. Equations (3) and (4) describe the gratings in Figs. $1 \mathrm{C}$ and $1 \mathrm{D}$ (termed here compound 1 and compound 2). Luminance $\left.\overline{(C}_{\text {lum }}\right)$ and chromatic $\left(C_{\text {chr }}\right)$ contrast can be controlled independently, although in the results presented here $C_{\text {lum }}$ and chromatic $C_{\mathrm{chr}}$ were set equal. Below each grating are waveforms representing the $R(\theta)$ and $G(\theta)$ gun modulations for a full stimulus cycle. The black, solid curve represents the luminance modulation in each stimulus (the mean of the red and green guns).

The gratings of Fig. 1C qualitatively resemble those in previous work [20], with the exception that a different, nonlinear function was used; the current function [Eq. (3)] is linear and more convenient for analysis and modeling of cell behavior. For the novel grating in Fig. $1 \mathrm{D}$, Compound 2A, the peak in luminance corresponded in phase to the maximum of the red gun. A half-cycle phase shift to the chromatic contrast term results in a different grating with the peak of luminance contrast corresponding to the maximum of the green gun (Compound 2B). Other phase relationships are also possible, resulting in a family of gratings with mixed luminance and chromatic composition, in which luminance and chromatic contrast can be separately manipulated. Equivalent grating sets can be generated targeting the $\mathrm{KC}$ ( $S$-cone) pathway, when the equiluminant components contain chromaticity lying along a tritanopic confusion line.

We consider here the relationship between psychophysical detection and discrimination thresholds of human observers to the physiological responses of macaque ganglion cells. Macaque retinal ganglion cell responsivity and response frequency spectra indicate that with these compound stimuli chromatic and luminance signals in the PC and MC pathways are clearly segregated at the physiological level. Psychophysical detection sensitivity of human observers for the compound grating types [Fig. $1 \mathrm{C}$ and $\underline{1 D}$ ] were compared with detection sensitivity for the chromatic and luminance gratings. Detection of compound gratings followed the envelopes of these latter curves, i.e., sensitivity corresponded to whichever (luminance or chromatic) curve had the highest sensitivity. A more critical test is provided by discrimination between compound gratings and luminance or chromatic gratings, which was possible over a broad range of spatial frequencies at detection threshold. These data strongly support a strict segregation of luminance and chromatic information in the afferent visual pathways with stimulus patterns of complex structure.

\section{METHODS}

\section{A. Physiological Recordings}

All procedures conformed to the National Institutes of Health Guide for the Care and Use of Laboratory Animals and were approved by the SUNY State College of Optometry Animal Care and Use Committee. Macaques (2 M. fascicularis, male $2.8-4.0 \mathrm{~kg}$ ) were initially sedated with an intramuscular injection of ketamine $(10 \mathrm{mg} / \mathrm{kg})$. Anesthesia was induced with sodium thiopental $(10 \mathrm{mg} / \mathrm{kg})$ and maintained with inhaled isoflurane $(0.2-2 \%)$ in a $70: 30 \mathrm{~N}_{2} \mathrm{O}-\mathrm{O}_{2}$ mixture. Local anesthetic was applied to points of surgical intervention. Electroencephalogram and electrocardiogram were monitored continuously to ensure animal health and adequate depth of anesthesia. Muscle relaxation was maintained by an infusion of gallamine triethiodide ( $5 \mathrm{mg} / \mathrm{kg} / \mathrm{hr}$ i.v.) with accompanying dextrose Ringer solution ( $5 \mathrm{ml} / \mathrm{kg} / \mathrm{hr}$ ). Body temperature was kept close to $37.5^{\circ} \mathrm{C}$. End-tidal $\mathrm{CO}_{2}$ was kept close to $4 \%$ by adjusting the rate and depth of respiration.

Neuronal activity was recorded directly from retinal ganglion cells by an electrode inserted through a cannula entering the eye behind the limbus. The details of the preparation can be found elsewhere [21]. A gas-permeable contact lens of the appropriate power was used to bring stimuli into focus on the retina. We recorded responses of cells between $4^{\circ}$ and $12^{\circ} \mathrm{ec}-$ centricity. Cell identification was achieved through standard tests [22]. These included achromatic contrast sensitivity and responses to lights of different chromaticity. PC retinal ganglion cells (midget ganglion cells of the parvocellular pathway) can generally be identified by their tonic responses and spectral opponency, and MC retinal ganglion cells (parasol ganglion cells of the magnocellular pathway) by their phasic responses and lack of spectral opponency. KC ganglion cells (e.g., small-bistratified cells of the koniocellular pathway) were identified by their characteristic response to $S$-cone isolating stimuli. For each cell, the locus of the receptive field center was determined and the stimulus was centered on 
this point. Times of spike occurrence in response to stimuli modulation were recorded to an accuracy of $0.1 \mathrm{~ms}$ with respect to the onset of a stimulus cycle, and averaged histograms of spike trains were accumulated with 64 bins per cycle of modulation. The histograms were Fourier analyzed to give amplitude and phase of the response spectra.

Responses to the different waveforms were measured using stimuli generated on a three-channel Maxwellian view system, which was controlled by a Macintosh Quadra 950 computer, as described elsewhere [23]. Briefly, a four primary stimulator was used [24], of which only two outputs were used in these experiments, with light-emitting diodes (LEDs) of dominant wavelengths of 638 and $554 \mathrm{~nm}$. These were collimated and combined to give a uniform field, and a field stop used to give a 4 deg. field. The LEDs were driven by pulse-train frequency modulation to achieve a highly linear relationship between driving voltage and LED intensity. They were usually modulated at $4.88 \mathrm{~Hz}$ at a time-averaged retinal illuminance close to $2000 \mathrm{Td}$.

\section{B. Psychophysical Methods}

The grating stimuli [Fig. 1] were generated via a VSG graphic controller (series 2/5, Cambridge Research Systems) and presented on a CRT monitor (Trinitron GDM-F500, $150 \mathrm{~Hz}$ frame rate). The gratings were presented in a circular window with a downward drift rate of $0.5 \mathrm{~Hz}$. Contrast sensitivity functions (CSFs) were compiled by varying the spatial frequency from 0.1 to 22.0 cycles per deg (cpd) by either changing the spatial frequency input to the VSG, or by increasing the subject's viewing distance. The viewing distance for $0.05-0.15 \mathrm{cpd}$ was $0.24 \mathrm{~m}$, for $0.1-1.5 \mathrm{cpd}$ it was $0.48 \mathrm{~m}$, for $1.5-6 \mathrm{cpd}$ it was $3.6 \mathrm{~m}$, and for $6-22$ cpd it was $7.2 \mathrm{~m}$. Additionally, to control for the effects of the subject's viewing distance, some frequencies were presented at both $0.24 \mathrm{~m}$ and $0.48 \mathrm{~m}, 0.48$ and $3.6 \mathrm{~m}$, as well as 3.6 and $7.2 \mathrm{~m}$. The mean luminance of all grating stimuli was $24.36 \mathrm{~cd} / \mathrm{m}^{2}$. The mean chromaticities were $(0.436,0.476)$ in CIE $X, Y$ coordinates for gratings intended to modulate a $M, L$-cone opponent channel, and $(0.200,0.161)$ for gratings targeted at an $S$-cone chromatic mechanism. The background surrounding the stimulus had a mean chromaticity and luminance the same as the target.

Observers. Psychophysical data were collected from six human observers. Observer BC is among the authors while observers KH, RE, and MJ were naïve consenting observers. Partial data sets were obtained from observers HS (one of the authors) and DW. All procedures conformed to the Declaration of Helsinki and the Association for research in vision ophthalmology, and all procedures were approved by the SUNY College of Optometry Institutional Review Board. Observers were shown to have normal color vision by the Farnsworth-Munsell 100-Hue Discrimination Test. BC, KH, and RE are myopic and wore corrective spectacles for all procedures. Each subject's isoluminance point was determined by the minimum motion technique [25] and this measurement was applied as individualized settings for the stimuli presented to each observer.

Procedure. We measured grating detection thresholds for all four stimuli [Fig. 1] as well as discrimination thresholds for the compound gratings against either the luminance or chromatic stimuli. Observers BC, KH, MJ, and RE completed full detection data sets for dichromatic (red/green) conditions, observers $\mathrm{BC}$ and $\mathrm{RE}$ completed all discrimination conditions, and observer BC completed a full data set with chromatic modulation along trichromatic ( $S$-cone) space. During either task, the observer viewed the stimulus foveally while gazing monocularly at a fixation cross.

During the detection task, the stimulus was shown in one of two successive temporal windows, each indicated with a beep, and the observer was asked to identify which interval contained the grating. During the discrimination task, there were again two temporal windows indicated with a beep. However, discrimination trials contained gratings in both intervals, one containing a compound grating and the other either the chromatic or luminance grating. The task was for the observer to identify which presentation contained a chromatic component (for discriminations between compound and luminance gratings) or a luminance component (for discrimination between compound and chromatic gratings).

The detection and discrimination thresholds were measured using a dual staircase procedure. A two-down, one-up procedure was used, with a step size of $0.3 \log$ unit, reduced to 0.15 log unit after the second reversal. Staircases were terminated after 12 reversals and the mean of the last six reversals used as a threshold estimate. With a dual staircase, this gave two thresholds, and the data shown represent means of 2-3 repetitions of the dual staircase.

\section{RESULTS}

\section{A. Physiological Recordings}

Neuronal activity was recorded to the waveforms in Fig. 1 using a uniform field (4 deg) stimulus. Responses were obtained from $16 \mathrm{MC}$ cells and $12 \mathrm{PC}$ cells. Characteristic response histograms are shown in Fig. $\underline{2}$ for four cell types (on- and off-center MC cells, and $+L-M$ and $+M-L$ PC cells), for $50 \%$ contrast, with the stimulus waveforms shown above the response histograms. In these and subsequent figures contrast refers to modulation contrast of the stimuli, rather than cone contrast related measures.

Figure 2A shows responses to luminance gratings. The PC cells deliver a variable luminance response and the MC cells respond strongly with on- and off-center cell response maxima occurring in counterphase to each other. The two PC cells give a vigorous response to the chromatic grating. The $L$ and $M$ cone polarity ( $L-M$ or $M-L)$ inverts the response phase [Fig. 2B]. The MC cells give a small frequency-doubled response [26]. Figures 2C, 2D, and 2E show responses to the compound stimuli (compound 1, and compound 2A,B; see Fig. 1). It can be seen that the PC cells' responses are dominated by the chromatic component of the stimulus. There are shifts in response phase and peak structure depending on the waveform, i.e., the $+L-M$ cell responds to the red parts of the gratings, and the $+M-L$ cell responds to the green parts of the grating. The MC cells' responses are dominated by the luminance component of the stimulus. There are two peaks for each MC cell in Fig. 2C, with the on- and off-center cells responding out of phase with one another, and one peak in Fig. $2 \mathrm{D}$ and $2 \mathrm{E}$, with the same phase in $\mathrm{D}$ and $\mathrm{E}$ for each cell.

Response histograms such as those in Fig. 2 were Fourier analyzed, and Fig. 3 shows Fourier spectra for the $+M-L$, $+L-M$ PC cells [Fig. 3A] and the on- and off-center MC cells [Fig. 3B]. There are larger first harmonic responses of PC 
A
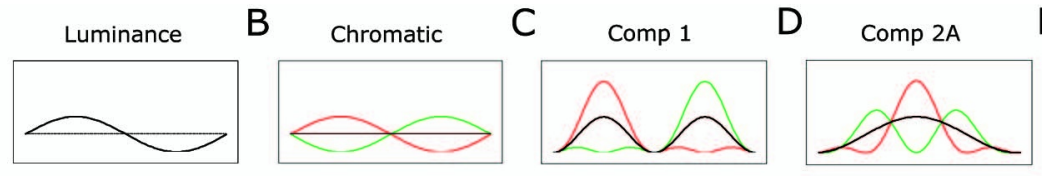

E Comp 2B
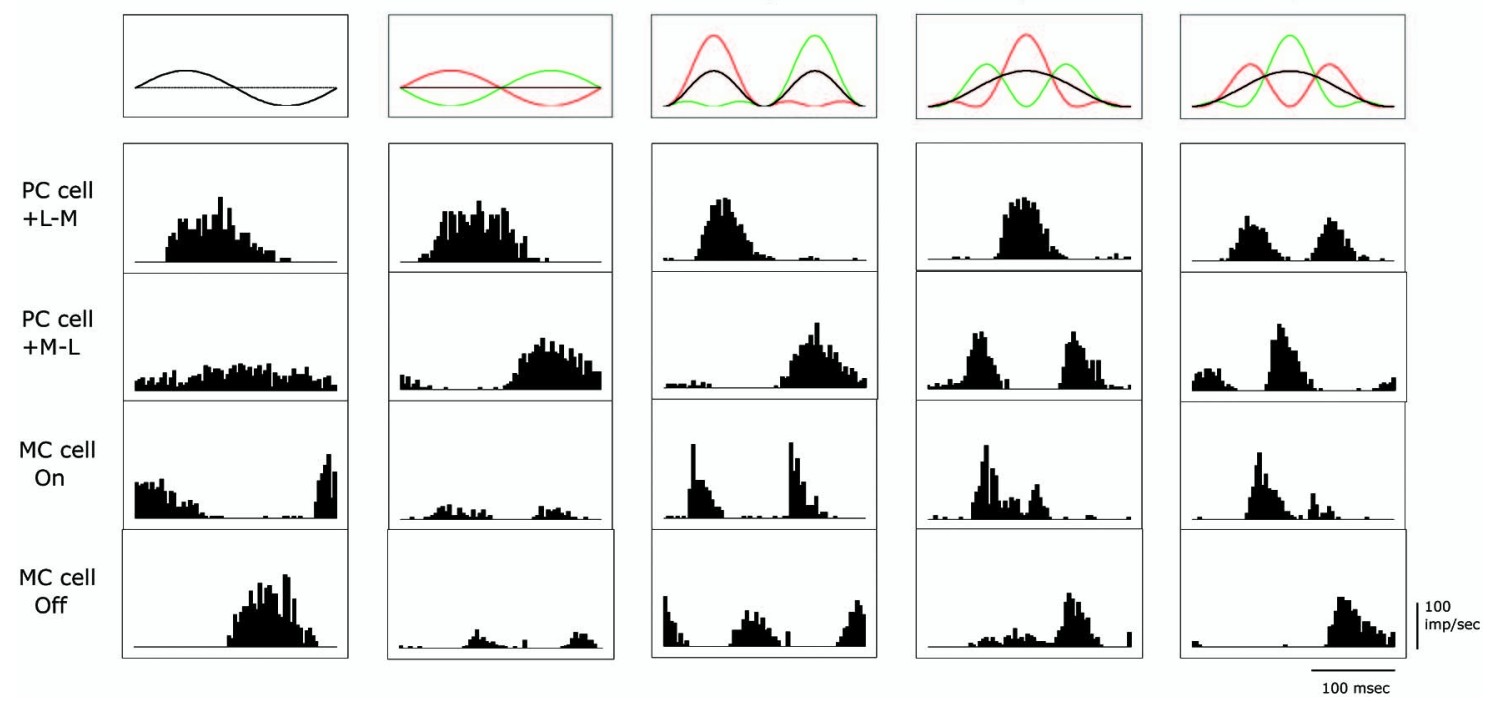

Fig. 2. Retinal ganglion cell response histograms. $+L-M$ and $+M-L \mathrm{PC}$ and on- and off-center MC Cell responses to grating stimuli. (A) Luminance, (B) Chromatic, (C) Compound 1, and (D and E) Compound 2 variants. $4^{\circ}$ stimuli were presented at $4.88 \mathrm{~Hz}$ using a Maxwellian view system: 50\% contrast. PC cells respond preferentially to the chromatic content of compound gratings whereas MC cells respond strongly to the luminance content. Average of 32 stimulus cycles, 64 bins/cycle.

cells to chromatic relative to luminance modulation. To the Compound 1 gratings, PC-cell first harmonic responses are dominant and to the Compound 2 gratings it is the second harmonic component (arrowed) that is dominant. For MC cells [Fig. 3B], first harmonic responses to luminance modulation are large but to chromatic modulation are almost absent. For compound 1 gratings the second harmonic (arrowed) is dominant, and for compound 2A,B the first harmonic is dominant. Phase behavior (not shown) showed a consistent pattern; for example, the second harmonic responses of $+L-M$ and $+M-L$ cells to the Compound 2 gratings were out-of-phase with respect to each other. It should be noted that there is energy in higher harmonics when the first harmonic response is large, but this was largely associated with response shaping, such as response rectification or a nonsinusoidal response waveform. These harmonic distortions decreased with contrast, as described with reference to Fig. 4 .

Responses to the different waveforms were measured as a function of contrast. Figure $4 \mathrm{~A}$ shows amplitudes of first and second harmonics as a function of contrast for luminance and chromatic modulation for the $+M-L \mathrm{PC}$ and on-center $\mathrm{MC}$ cells. The $+L-M$ PC and off-center MC showed similar patterns (data not shown). For the PC cell, the second harmonic response becomes negligible at low contrast, which is consistent with the response waveform approximating a sinusoid about the maintained firing level at these low contrasts. The same holds for the MC cell and luminance modulation; the MC cell shows a dominant second harmonic response to chromatic modulation, as described previously [26]. In Fig. 4B, responses to the compound 1 grating are plotted. For the $\overline{\mathrm{PC}}$-cell the first harmonic is dominant and the second harmonic is small at low contrast. For the MC cell, the reverse is the case. Similarly, in Fig. 4B for the compound 2 grating, the second harmonic responses of the PC cell are dominant with weak first harmonic responses at low contrast; the reverse is the case for the MC cell. This indicates that the selectivity of $\mathrm{PC}$ and MC cells for chromatic and luminance components of compound gratings is most marked at low contrast, as is the case near psychophysical detection threshold.

Data shown in Figs. $\underline{3}-\underline{4}$ were typical of the cell sample. At $50 \%$ contrast, the first/second harmonic ratios for PC cells averaged 2.2 [standard deviation (s.d.) $0.75, n=12$ ] for compound 1, and 0.47 (s.d. 0.31) for compound 2 gratings. For MC cells, the ratios were 0.22 (s.d. $0.16, n=16$ ) and 2.8 (s.d. 1.24). There was some intercell variability; 3 off-center PC cells showed ratios greater than one for compound 2 gratings, and some MC cells showed higher harmonic distortions with compound gratings at high contrast. This might be related to the nonlinear, frequency-doubled response of these cells to chromatic modulation. These estimates confirm the consistency of the results over the populations of cells.

The contrast data in Fig. $\underline{4}$ are shown to demonstrate that PC and MC cell signals with compound waveforms show considerable specificity for chromatic and luminance components, respectively; in the next section, it is shown that detection and discrimination of compound versus luminance and chromatic gratings is possible near detection threshold, and the physiological data strongly suggest that this requires comparison of PC and MC cell activity.

\section{B. Psychophysical Thresholds}

Luminance and chromatic CSFs were first measured [Fig. 1A and $1 \mathrm{~B}]$ to provide templates for comparison with psychophysical performance on detection and discrimination tasks of compound gratings [Fig. 1C and 1D]. Figure 5A shows CSFs for luminance and chromatic gratings of two human observers; the curves show characteristic bandpass and lowpass shapes, respectively. Luminance sensitivity is maximal in a range at approximately $0.8-4$ cpd whereas chromatic sensitivity is greatest at low spatial frequencies, as in previously published results [27]. It should be noted that we did not correct for chromatic aberration and, consequently, data points at spatial frequencies above $6 \mathrm{cpd}$ were not used in the fits described below. 
A

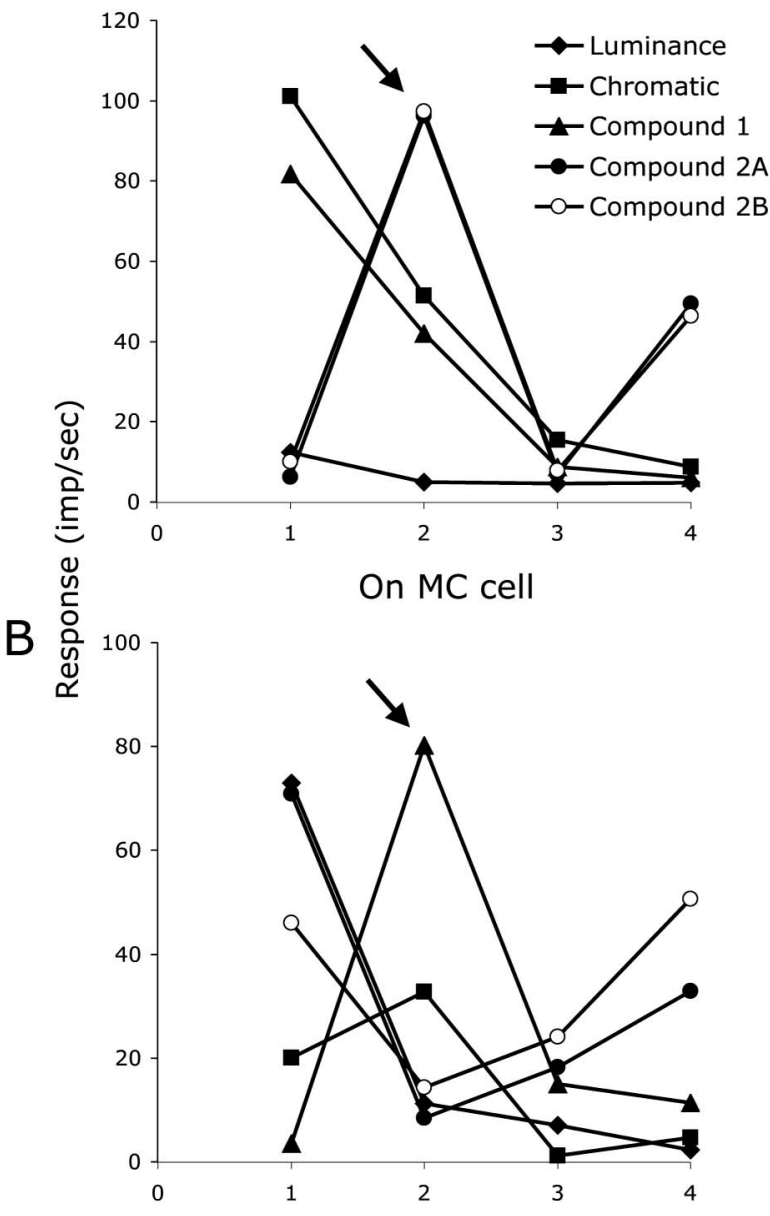

$+\mathrm{L}-\mathrm{M}$ PC cell
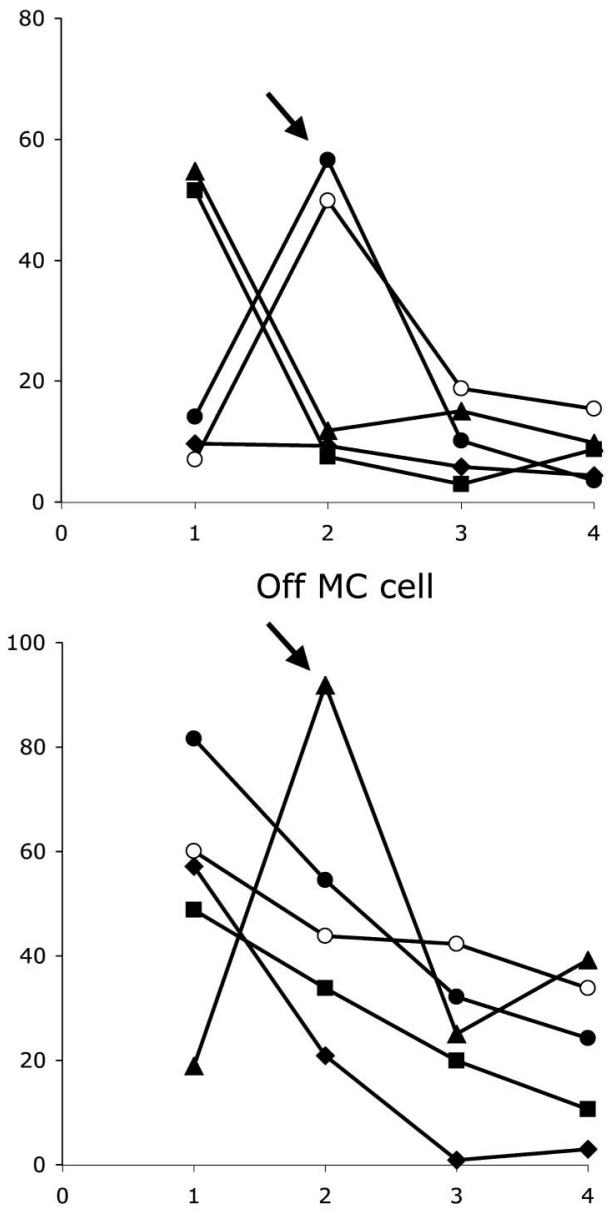

Harmonic

Fig. 3. Harmonic composition of PC and MC cell responses. Fourier spectra for (A) the $+M-L,+L-M$ PC cells and (B) the on- and off-center MC cells of Fig. 2. PC cells exhibit strong responses to the chromatic content in compound gratings with the majority of energy in the first harmonic for compound $\overline{1}$ and the second harmonic for compound 2 (Compounds $2 \mathrm{~A}, 2 \mathrm{~B}$ ). The reverse is true for MC cells. MC cells respond chiefly to the luminance content of compound gratings with the majority of MC energy localized in the second harmonic of compound 1 (Compound 1 ) and the first harmonic of compound 2 (Compounds 2A,2B).

We fitted the luminance and chromatic contrast sensitivity functions with filter models; a lowpass 2-stage filter [Eq. (5)] for the chromatic red-green contrast sensitivity function ( $S_{\text {chrom }}$ ), and a difference of a 3-stage and 1-stage lowpass filter [Eq. (6)] for the luminance contrast sensitivity function $\left(S_{\text {lum }}\right)$.

$$
\begin{gathered}
S_{\text {chrom }}=\frac{k_{0}}{\left(1+2 \pi\left(f / f_{0}\right)^{2}\right)^{\frac{2}{2}}} \\
S_{\text {lum }}=\frac{k_{1}}{\left(1+2 \pi\left(f / f_{1}\right)^{2}\right)^{\frac{3}{2}}}-\frac{k_{2}}{\left(1+2 \pi\left(f / f_{2}\right)^{2}\right)^{\frac{1}{2}}},
\end{gathered}
$$

where $f$ is spatial frequency in cycles/deg, $f_{0}, f_{1}, f_{2}$ are corner frequencies, and $k_{0}, k_{1}$, and $k_{2}$ are free scaling factors; use of such filter models is common [28]. The fit results are shown as dashed (chromatic CSF) and solid (luminance CSF) lines in Fig. 5. For the six observers, the corner frequency for the equiluminant red-green curve, $f_{0}$, ranged from 0.25 to 0.45 , and two corner frequencies for the luminance curve, $f_{1}$ and $f_{2}$, varied from 0.04 to 0.05 and from 1.1 to $1.4 \mathrm{cpd}$, respectively.

These curves were then used as templates in relation to observers' sensitivity to compound gratings to ascertain which mechanism might be responsible. Spatial frequency of the templates was adjusted as necessary. For instance, the luminance template for compound 1 is shifted laterally by a factor of 0.5 on the $x$ axis since the luminance grating is doubled in frequency. For compound 2, the chromatic template is shifted laterally by a factor of 0.5 on the $x$ axis since the chromatic grating is doubled in frequency. Additionally, the chromatic templates are adjusted vertically to account for small differences in root-mean-square (RMS) chromatic contrast in the different gratings (along the $y$ axis by a factor of 1.78 for compound 1 and by 1.58 for compound 2 [20]).

With these adjustments to the templates a comparison can be made to determine the contribution luminance and chromatic detection mechanisms make to the detection of compound gratings. The detection of compound 1 and compound 2 [Fig. $5 \mathrm{~B}$ and $5 \mathrm{C}$ ] follows the envelopes of the luminance and chromatic CSF over spatial frequency. For low spatial frequencies, sensitivity to compound gratings matches the chromatic CSF as this mechanism is most sensitive in this range. At higher spatial frequencies observers' detection of compound gratings matches luminance CSF more closely. 

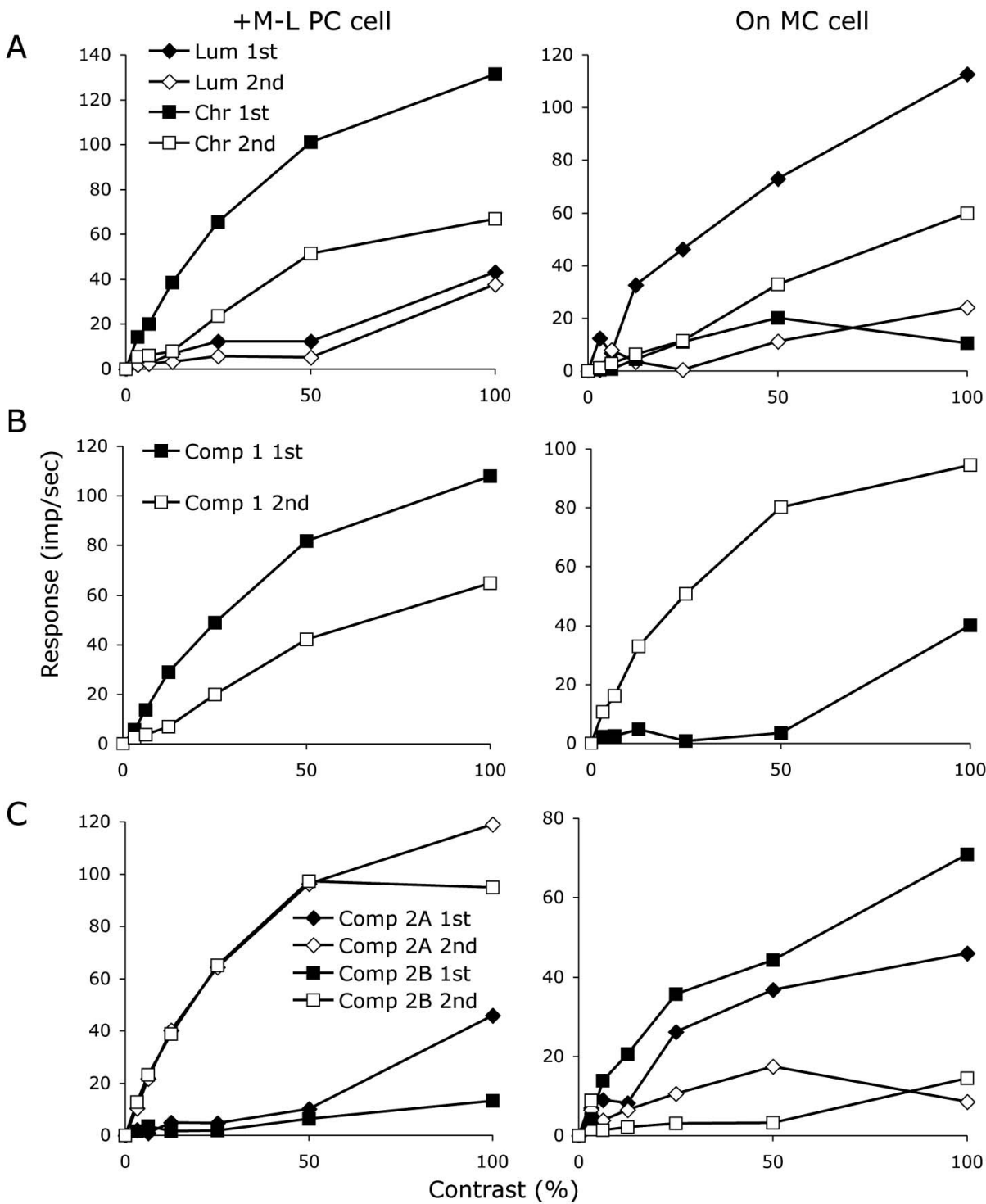

Fig. 4. Harmonic composition of $\mathrm{PC}$ and MC cells as a function of contrast. First and second harmonic composition of an $+M-L \mathrm{PC}$ cell and an On MC cell for (A) standard luminance and chromatic modulation, (B) Compound 1, (C) both variations of Compound 2. The PC and MC response to compound gratings is again specific to either the chromatic or luminance content respectively, and this relation is maintained across a wide range of contrast.

Similar data sets were obtained from the other observers [Fig. 5 shows two examples of complete detection data sets from the six observers tested]. The detection results are consistent with the hypothesis that luminance and chromatic signals are carried in separate pathways. However, the chromatic and luminance templates overlap to a considerable degree and it could be argued that the segregation of luminance and chromatic templates is not large enough to draw firm conclusions. We therefore performed discrimination experiments that provide stronger evidence for segregation of afferent signals.

Based on the physiological analysis in the previous section, chromatic and luminance response components of compound waveforms are well segregated in the PC and MC pathways, and this remains so at low contrast. We hypothesized that if observers can distinguish compound gratings from luminance gratings near detection threshold, then this is consistent with independent activation of the two pathways. For example, if the task was to discriminate between a luminance and a com- pound grating presented in two sequential intervals, the observer had to distinguish in which of the two intervals a chromatic component was present. Conversely, in the case of discrimination of the compound grating from the chromatic grating, the observer has to say which interval contained a luminance signal. For these discrimination experiments, the contrast of the two gratings was always the same, and the staircase procedure determined the threshold contrast at which the gratings could be discriminated. Figure 6 shows performance for the two observers at discrimination tasks in which the observer must distinguish a compound grating from either the luminance (A, C) or the chromatic (B, D) grating. The detection templates have been drawn in as before. The results in Fig. 6 indicate that the observer is capable of performing the discrimination task at detection threshold under almost all conditions.

We suggest that the discrimination task is a more stringent demonstration of the independence of luminance and chromatic mechanisms because the observer must respond to a 

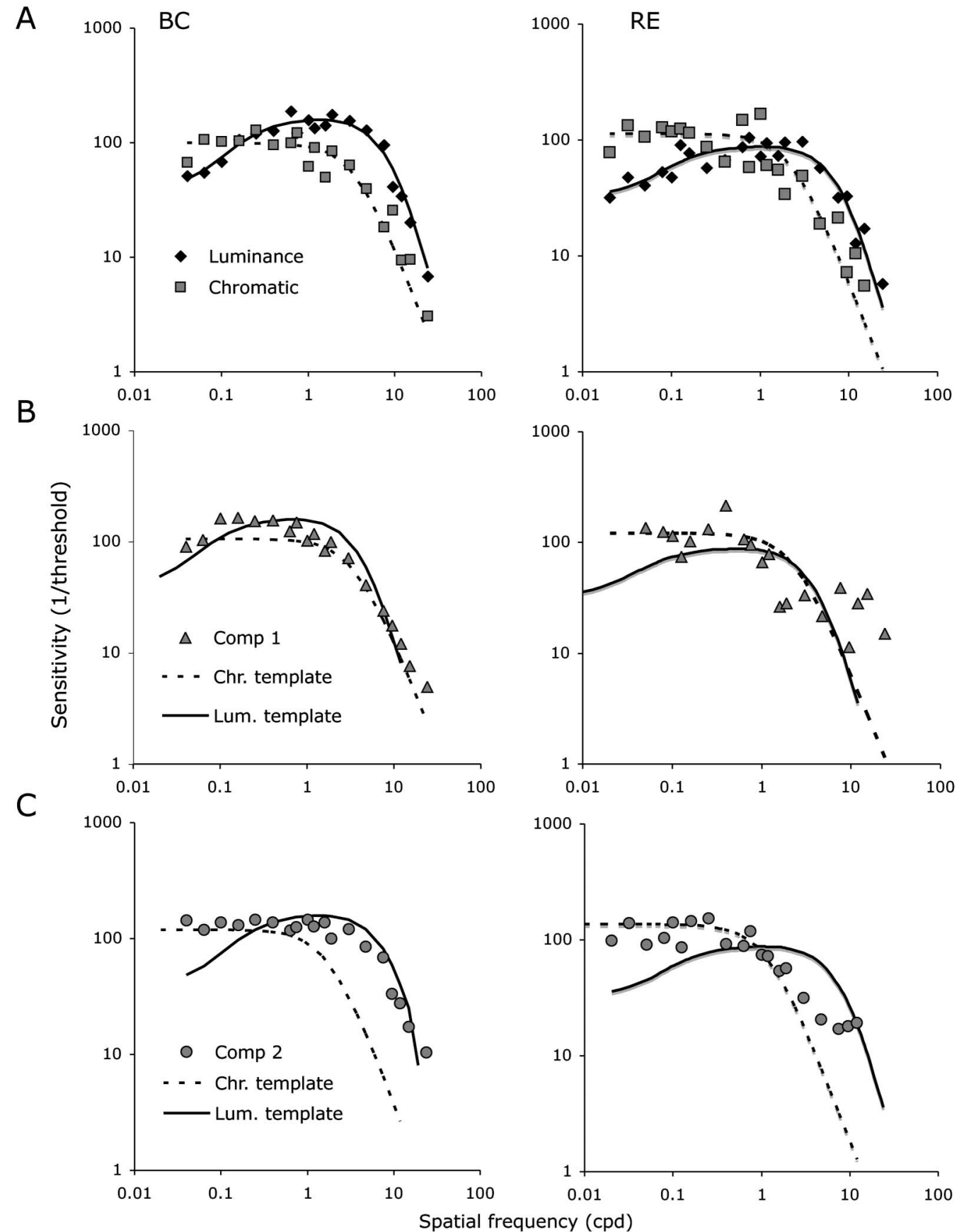

Fig. 5. Luminance and chromatic spatial contrast sensitivity functions and detection thresholds of compound grating types. Detection thresholds are plotted for observers BC and RE: (A) Solid and dashed curves represent filter fits to the observer's detection thresholds for luminance and redgreen isoluminant chromatic modulation. The luminance CSF consists of a bandpass filter that is a difference between a 3-stage and 1-stage lowpass filter. Chromatic CSFs are fit with a lowpass 2-stage filter. (B) CSFs and detection thresholds of Compound 1. The luminance curve [filter fit from (A)] was shifted along the $x$-axis by a factor of 0.5 to account for relative spatial frequency of the luminance content, and the chromatic CSF was shifted by a factor of 1.78 along the $y$-axis to account for the relative RMS chromatic contrast of the chromatic and compound gratings. (C) CSFs and detection thresholds of Compound 2 . The chromatic curve was shifted along the $x$-axis by a factor of 0.5 to account for relative spatial frequency of the chromatic content and was shifted by a factor of 1.58 along the $y$-axis to account for the relative chromatic contrast of the chromatic and compound gratings. The chromatic CSF is more sensitive at low spatial frequencies whereas the luminance CSF dominates at higher spatial frequencies. Therefore, the detection of compound gratings appears to be mediated by the mechanism that is most sensitive at a particular spatial frequency.

specific waveform component of the compound grating whereas, for the detection task, the observer is only indicating the presence of a stimulus. The sensitivity should follow the profile of the CSF of the mechanism making the discrimination. This was generally the case for the two observers shown, and was also the case for three other observers tested.
This is most prominent in the results for Compound 2 [Fig. 6C and 6D]. Data for the discrimination of Compound 2 from luminance modulation follows the envelope of the chromatic CSF because the chromatic component is the distinguishing feature of the compound grating in that condition, and vice versa in the compound compared to 

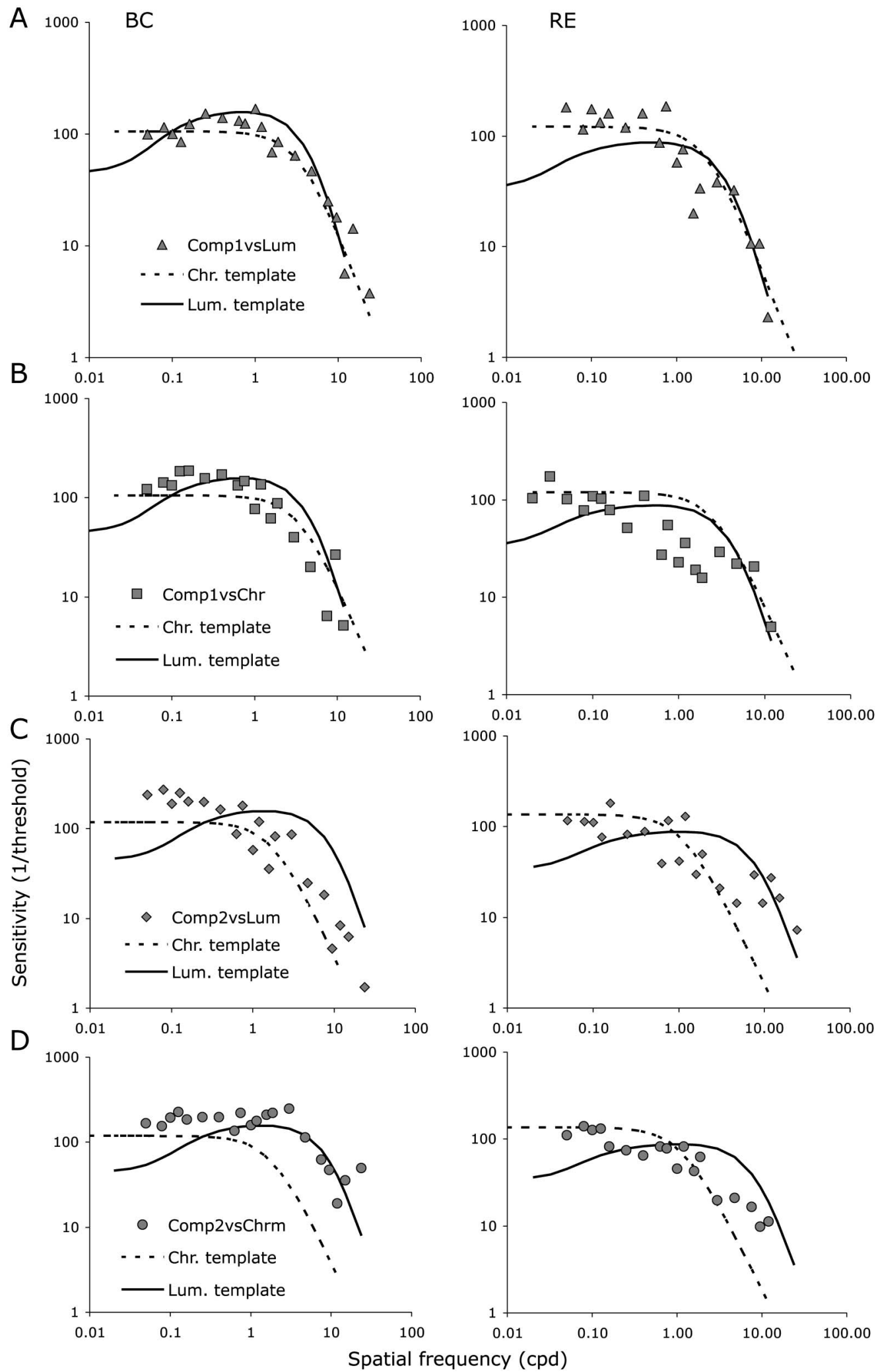

Fig. 6. Psychophysical thresholds of the discrimination of compound gratings from luminance and chromatic gratings. Thresholds for observers $\mathrm{BC}$ and RE for the conditions (A) Compound 1 discrimination from a luminance grating, (B) Compound 1 discrimination from a chromatic grating, (C) Compound 2 discrimination from a luminance grating, and (D) Compound 2 discrimination from a chromatic grating. The discrimination task is robust down to threshold, which is consistent with independent luminance and chromatic mechanisms permitting discrimination.

chromatic condition. These results suggest that chromatic and luminance mechanisms are independently utilized for discrimination.
We also tested this protocol with gratings designed to selectively activate $S$-cone pathways. The goal was to determine if a similar segregation of luminance and chromatic spatial 

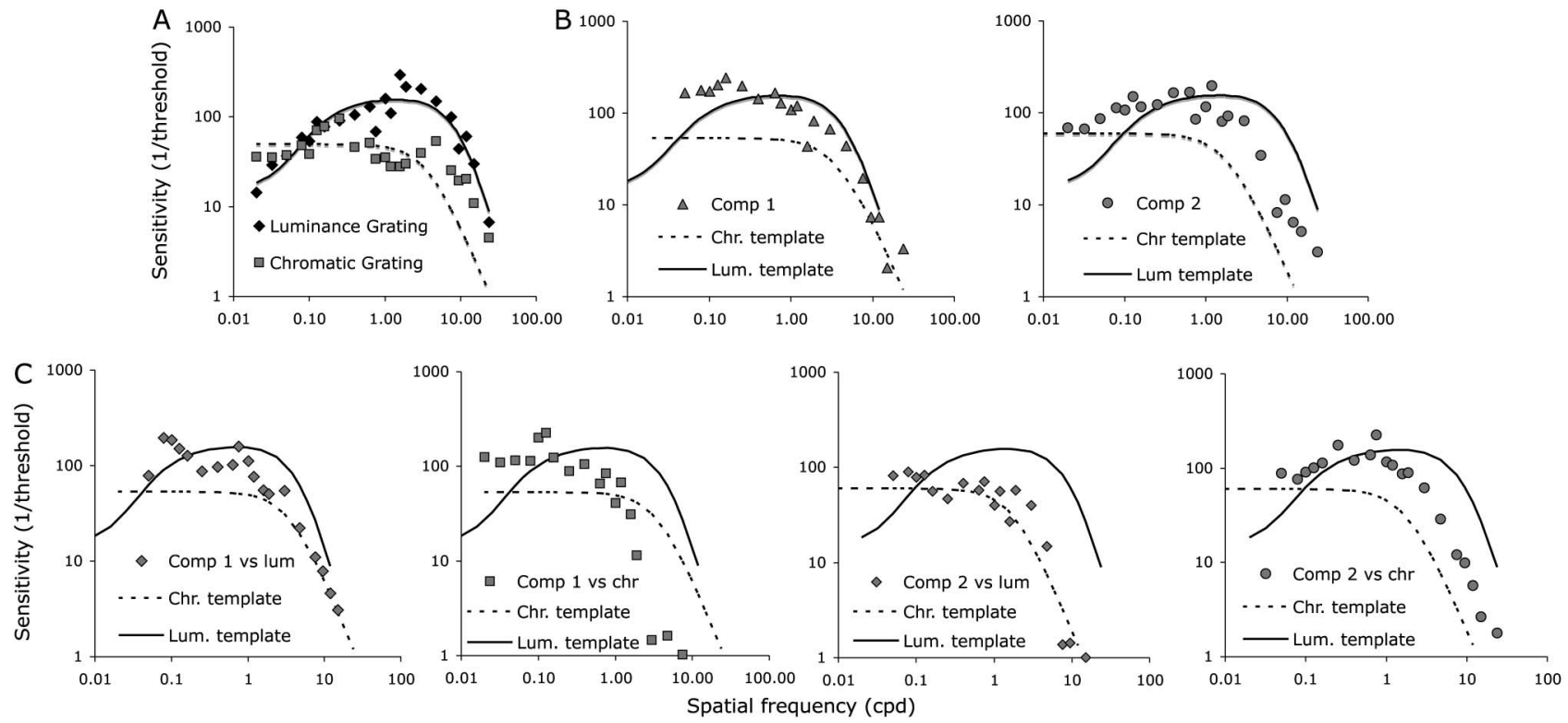

Fig. 7. Tritan psychophysical spatial contrast sensitivity functions of different grating types. Detection and discrimination thresholds for observer BC: (A) Luminance and blue-yellow tritan contrast sensitivity functions. Filter fits applied to B-Y results are analogous to the R-G CSF models. (B) Detection of compound 1 and compound 2 gratings. As with R-G modulation, B-Y detection follows the envelope of the more sensitive CSF. (C) Discrimination data for both compounds 1 and 2 from luminance and chromatic gratings. The same adjustment for the luminance and chromatic CSFs regarding relative spatial frequency and RMS chromatic contrast were used as for red-green detection and discrimination.

information might occur along a blue-yellow chromatic dimension. Detection and discrimination thresholds were obtained for conditions similar to those used for red-green gratings, except for a different mean chromaticity (see methods). Figure 7A shows detection thresholds and fitted curves for luminance and chromatic gratings and Fig. 7B the detection thresholds for compound waveforms. Detection CSFs of compound gratings follow the envelope of the more sensitive mechanism (either standard luminance or chromatic CSF) at a given spatial frequency. For the discrimination task, thresholds were usually close to detection threshold although under some conditions at higher spatial frequencies thresholds are lower than the template. Similar data were obtained from two other observers. These results lend further support for the transmission of luminance and chromatic signals via independent pathways.

\section{DISCUSSION}

Psychophysical CSFs for chromatic and achromatic gratings differ. This is thought to be due to different spatial properties of parallel chromatic and achromatic pathways in vision [27,29]. Interactions between chromatic and achromatic spatial channels has been studied, for example in cross-masking experiments [30], but the compound gratings we present here are an alternative means of studying their interaction. We suggest that this approach is relevant to studies of the natural environment, which commonly contains variegated colored areas interspersed with shade. The previously detailed equations (1), (2), (3), and (4) allow independent manipulation of the luminance and chromatic contrast, spatial frequency, and phase to study a diverse variety of waveforms. We have presented here physiological and psychophysical results for a limited set of these permutations.

$\mathrm{MC}$ and PC response histograms to the compound gratings [Fig. 2] strongly support a physiological segregation of lumi- nance and chromatic information at the retinal level. Although the current physiology data were only derived from uniform field stimuli, an earlier paper shows the conclusion to hold over a very broad range of spatial frequencies [20]. MC cells respond selectively to luminance and PC cells to chromatic grating components. Generally PC cells yield weak responses to luminance as with the $|+M-L| \mathrm{PC}$ cell of Fig. 2A. On occasion, $\mathrm{PC}$ cells may give a stronger luminance signal, as did the $|+L-M| \mathrm{PC}$ cell in Fig. 2A. However, the response to compound gratings from this $\overline{\mid}+L-M \mid \mathrm{PC}$ cell is still dominated by the chromatic component of the waveform [Fig. 3]. Thus, PC cells exhibited responses to compound gratings that were dominated by the chromatic content of compound gratings regardless of the response to luminance modulation alone. Our results suggest that the contribution of a PC cells response to compound gratings and, by proxy natural scenes, is a selective chromatic signal.

The concept of multiplexing chromatic and luminance information in the PC pathway [8] calls for PC neurons to deliver an achromatic signal at high spatial frequencies and a subtractive, $L, M$ opponent signal at low spatial frequencies. This hypothesis rests on the assumption that PC cells have bandpass achromatic tuning curves because of center surround structure (Type 1 in Wiesel and Hubel's nomenclature [31]). While some PC cell achromatic tuning curves may show a bandpass characteristic to luminance gratings [32], it has been shown that there is much variability within the cell population [33], as can be seen in the data of Derrington et al. [32] Many cells show little or no bandpass property. In the physiology results with compound gratings presented here, the relative energy distribution of the Fourier spectra for a $\mathrm{PC}$ cell's response is consistently found in the same harmonic as the chromatic component of either compound waveform [Fig. 3: first harmonic in compound 1 and second harmonic of compound 2]. There is much less energy in the luminance 
component of the PC response, and therefore it appears unlikely that the response could carry meaningful information regarding the luminance structure of the compound grating. On the other hand, there is a vigorous representation of the luminance spatial signal mediated by the MC pathway.

Our results are consistent with the point of view that independent luminance and chromatic signals are an efficient means of transmitting visual information [4] in comparison to a multiplexed signal in the PC pathway. This former suggestion was extended by MacLeod and his colleagues [5,6] who considered noise in neuronal signals and the advantages of dividing, say, cells of the PC pathway into $+M-L$ and $+L-M$ types (split-range coding). These arguments should hold irrespective of spatial frequency, unlike the prediction of the double-duty hypothesis.

There is evidence that in the cortex the coding of color and luminance is less well segregated than in the afferent pathways [34]; addition of PC and MC inputs have been suggested based on the physiological responses of V1 double opponent color-luminance cells [35]. However, from our data detection, sensitivities to compound gratings followed the envelope of luminance and chromatic CSF values and the discrimination of compound versus luminance and chromatic gratings near threshold is evidence of the discrimination being performed through independent achromatic and chromatic channels, requiring comparison of PC and MC cell activity. For example, when distinguishing compound gratings from luminance gratings, both contain a luminance component and presumably the observer detects which grating contains a chromatic component (and the other way round for discrimination of compound and chromatic gratings). This implies separation of MC and PC cells is to some degree maintained centrally. However, this issue remains unresolved.

\section{ACKNOWLEDGMENTS}

This work was supported by the National Eye Institute (NEI) (EY13112).

\section{REFERENCES}

1. C. A. Parraga, G. Brelstaff, T. Troscianko, and I. R. Moorehead, "Color and luminance information in natural scenes," J. Opt. Soc. Am. A 15, 563-569 (1998).

2. T. Wachtler, T. Lee, and T. J. Sejnowski, "Chromatic structure of natural scenes,” J. Opt. Soc. Am. A 18, 65-77 (2001).

3. G. J. Burton and I. R. Moorhead, "Color and spatial structure in natural scenes,” Appl. Opt. 26, 157-170 (1987).

4. G. Buchsbaum and A. Gottschalk, "Trichromacy, opponent colours coding and optimum colour information transmission in the retina," Proc. R. Soc. B 220, 89-113 (1983).

5. T. von der Twer and D. I. MacLeod, "Optimal nonlinear codes for the perception of natural colors," Network 12, 395-407 (2001).

6. D. I. A. MacLeod and T. von der Twer, "The pleistochrome: optimal opponent codes for natural colours," in Color Perception: Mind and the Physical World, R. Mausfeld and D. Heyer, eds. (Oxford University, 2003).

7. H. B. Barlow, "What causes trichromacy? A theoretical analysis using comb-filtered spectra," Vis. Res. 22, 635-643 (1982).

8. C. R. Ingling and E. Martinez-Uriegas, "The relationship between spectral sensitivity and spatial sensitivity for the primate $\mathrm{r}-\mathrm{g}$ X channel," Vis. Res. 23, 1495-1500 (1983).

9. P. Lennie and M. D. D'Zmura, "Mechanisms of color vision," CRC Crit. Rev. Neurobiol. 3, 333-400 (1988)
10. A. M. Derrington, J. Krauskopf, and P. Lennie, "Chromatic mechanisms in lateral geniculate nucleus of macaque," J. Physiol. 357, 241-265 (1984).

11. F. A. A. Kingdom and K. T. Mullen, "Separating colour and luminance information in the visual system," Spatial Vision 9, 191-219 (1995).

12. E. Kaplan, B. B. Lee, and R. M. Shapley, "New views of primate retinal function," Progr. Retinal Res. 9, 273-336 (1990).

13. R. M. Shapley, "Visual sensitivity and parallel retinocortical channels," Annu. Rev. Psych. 41, 635-658 (1990).

14. B. B. Lee, "Visual pathways and psychophysical channels in the primate," J. Physiol. 589, 41-47 (2011).

15. B. B. Lee, P. R. Martin, and A. Valberg, "The physiological basis of heterochromatic flicker photometry demonstrated in the ganglion cells of the macaque retina," J. Physiol. 404, 323-347 (1988).

16. D. M. Dacey, "Circuitry for color coding in the primate retina," Proc. Natl. Acad. Sci. USA 93, 582-588 (1996).

17. C. Tailby, S. G. Solomon, and P. Lennie, "Functional asymmetries in visual pathways carrying $S$-cone signals in macaque," J. Neurosci. 28, 4078-4087 (2008).

18. F. W. Campbell and J. G. Robson, "Application of Fourier analysis to the visibility of gratings,” J. Physiol. 197, 551-566 (1968).

19. J. Nachmias and A. Weber, "Discrimination of simple and complex gratings," Vis. Res. 15, 217-223 (1975).

20. B. B. Lee, H. Sun, and A. Valberg, "Segregation of chromatic and luminance signals using a novel grating stimulus," J. Physiol. 589, 59-73 (2011).

21. J. M. Crook, B. Lange-Malecki, B. B. Lee, and A. Valberg, "Visual resolution of macaque retinal ganglion cells," J. Physiol. 396, 205-224 (1988).

22. B. B. Lee, P. R. Martin, and A. Valberg, "Sensitivity of macaque retinal ganglion cells to chromatic and luminance flicker," J. Physiol. 414, 223-243 (1989).

23. B. B. Lee, J. Pokorny, V. C. Smith, P. R. Martin, and A. Valberg, "Luminance and chromatic modulation sensitivity of macaque ganglion cells and human observers," J. Opt. Soc. Am. A 7, 2223-2236 (1990).

24. J. Pokorny, H. Smithson, and J. Quinlan, "Photostimulator allowing independent control of rods and the three cone types," Vis. Neurosci. 21, 263-267 (2004).

25. S. Anstis and P. Cavanagh, "A minimum motion technique for judging equiluminance," in Colour Vision Physiology and Psychophysics, J. D. Mollon and L. T. Sharpe, eds. (Academic, 1983), pp. 155-166.

26. B. B. Lee, P. R. Martin, and A. Valberg, "Nonlinear summation of $M$ - and $L$-cone inputs to phasic retinal ganglion cells of the macaque," J. Neurosci. 9, 1433-1442 (1989).

27. K. T. Mullen, "The contrast sensitivity of human colour vision to red-green and blue-yellow chromatic gratings," J. Physiol. 359, 381-400 (1985).

28. A. B. Watson, "Temporal Sensitivity," in Handbook of Perception and Human Performance, K. R. Boff, L. Kaufman, and J. P. Thomas, eds. (Wiley, 1986), pp. 6-1-6-43.

29. R. L. DeValois and K. K. DeValois, eds., Spatial Vision, Oxford Psychology Series (Oxford University, 1988).

30. E. Switkes, A. Bradley, and K. K. De Valois, "Contrast dependence and mechanisms of masking interactions among chromatic and luminance gratings," J. Opt. Soc. Am. A 5, 1149-1162 (1988).

31. T. Wiesel and D. H. Hubel, "Spatial and chromatic interactions in the lateral geniculate body of the rhesus monkey," J. Neurophysiol. 29, 1115-1156 (1966).

32. A. M. Derrington and P. Lennie, "Spatial and temporal contrast sensitivities of neurones in lateral geniculate nucleus of macaque," J. Physiol. 357, 219-240 (1984).

33. B. B. Lee, R. M. Shapley, M. J. Hawken, and H. Sun, "Spatial distribution of cone inputs to cells of the parvocellular pathway," J. Opt. Soc. Am. A 29, A223-A232 (2012).

34. K. R. Gegenfurtner, "Cortical mechanisms of color vision," Nat. Rev. Neurosci. 4, 563-572 (2003).

35. E. N. Johnson, M. J. Hawken, and R. Shapley, "Cone inputs in macaque primary visual cortex,” J. Neurophysiol. 91, 2501-2514 (2004). 